Ambiente \& Água - An Interdisciplinary Journal of Applied Science
ISSN 1980-993X - doi:10.4136/1980-993X
www.ambi-agua.net
E-mail: ambi.agua@gmail.com

\title{
Reservas legais: um importante refúgio para os isópodas terrestres em áreas agrícolas
}

\author{
doi: 10.4136/ambi-agua.1476
}

Received: 03 Aug. 2014; Accepted: 21 Mar. 2015

\author{
Noeli Júlia Schüssler de Vasconcellos ${ }^{1 *}$; Rodrigo Ferreira da Silva ${ }^{2}$; \\ Fernando Henrique Bins ${ }^{1}$; Bruna Leite da Silva ${ }^{1}$ \\ ${ }^{1}$ Centro Universitário Franciscano (Unifra), Santa Maria, RS, Brasil \\ Curso de Engenharia Ambiental e Sanitária \\ ${ }^{2}$ Universidade Federal de Santa Maria (UFSM), CESNORS, Frederico Westphalen, RS, Brasil \\ *Autor correspondente: e-mail: noejuabio@yahoo.com.br, \\ rodrigosilva@smail.ufsm.br,fernando_bins@hotmail.com, brunaleitte@ hotmail.com
}

\section{RESUMO}

Isópodas terrestres (Crustacea) são macro decompositores de matéria vegetal morta e bioacumuladores de metais. $\mathrm{O}$ manejo inadequado do solo tem alterado a dinâmica das populações de isópodas, comprometendo o equilíbrio dos agroecossistemas. Foi investigado o potencial das áreas de mata preservada como refúgio para as populações de isópodas em agroecossistemas. A investigação foi realizada em áreas de cultivo de feijão, milho, azevem e soja e áreas de mata preservada adjacente. Foram amostrados três pontos aleatórios em cada cobertura vegetal, por meio de quadrados de $40 \mathrm{~cm} \mathrm{x} 40 \mathrm{~cm}$, nos quais se coletou toda serapilheira de mata e $0-10 \mathrm{~cm}$ de solo. Paralelamente foi determinada matéria orgânica, $\mathrm{pH} \mathrm{e}$ conteúdo de água no solo. Os isópodas foram removidos das amostras, contados e conservados. Foram encontrados 43 exemplares nas matas preservadas e nenhum nos cultivos. Apenas duas espécies foram identificadas. Os resultados obtidos evidenciam que o impacto nas áreas agrícolas altera o habitat e o desenvolvimento dos isópodas e que as áreas de mata preservada podem constituir um importante refúgio esse grupo de organismos.

Palavras-chave: área cultivada, floresta nativa preservada, isópodas, solo.

\section{Legal reserves: an important refuge for terrestrial isopods in agricultural areas}

\begin{abstract}
Terrestrial isopods (Crustacea) are macro decomposers of organic matter and bio-accumulators of metals. Soil management and farming practices have changed the population dynamics of isopods, jeopardizing the balance of agro ecosystems. We investigated the potential of preserved forest areas as a refuge for isopod populations in agro-ecosystems. The investigation was carried out in areas with different cover types: beans, corn, soybeans, rye grass and adjacent preserved native forest areas. We sampled three points at random using squares of $40 \mathrm{~cm} \mathrm{x} 40 \mathrm{~cm}$, in which we collected the litter of the forest area and a $0-10 \mathrm{~cm}$ layer of soil. We also estimated the organic matter, $\mathrm{pH}$ and soil moisture. The isopods were removed from the samples, counted and preserved. Forty-three specimens were
\end{abstract}


found in the forest samples and none in the samples from cultivated areas. Only two distinct species were identified. The results showed the impact of agricultural areas on populations of terrestrial isopods, and the importance of preserved forest areas as a refuge for isopods.

Keywords: cultivated area, isopod, preserved native forest, soil.

\section{INTRODUÇÃO}

Os Isopodas da subordem Oniscidea são considerados os primeiros crustáceos verdadeiramente terrestres (Rupert et al., 2005). Pertencem a mesofauna endógena do solo medindo 1,2 a 30 mm (Furlan, 1996; Paoletti e Hassal, 1999) e desempenham um importante papel no meio ambiente, atuando na ciclagem de nutrientes. São considerados seres saprófagos detritívoros, por triturar e reduzir a serrapilheira (resíduos da vegetação) em fragmentos menores, ação essa fundamental para as atividades dos microrganismos no solo.

Os isópodas podem assimilar grande quantidade de todo resíduo vegetal de uma área, o que comprova a importância desses organismos no processo de decomposição primária da matéria orgânica (Grünwald, 1988). Eles também atuam no transporte de propágulos de bactérias, fungos e micorriza arbuscolar vesiculosa através dos solos (Rabatin e Stinner, 1988; Caseiro et al., 2000). São classificados conforme sua ecomorfologia em Corredores, Enroladores e Aderentes (Paoletti e Hassall, 1999).

De acordo com Garcia e Campos (2001) os crustáceos terrestres conseguiram adaptar-se em ambiente terrestre pela substituição das brânquias por estruturas respiratórias parecidas com pulmões, denominados pulmões pleopodais ou pseudotraquéas, responsáveis pelas trocas gasosas (Furlan, 1996). Algumas espécies atraqueadas mantiveram as brânquias, e foram classificados como crustáceos terrestres atraqueados umícolas, dependentes de lugares com considerável umidade no solo para a respiração (Furlan, 1996). A água para sedentação é obtida em grande parte pelo alimento consumido, e ou por ingestão direta com o auxílio das peças bucais, em ocasiões de estresse hídrico existem cavidades corporais conhecidas como urópodes que tocam o solo e absorve a umidade do ambiente por meio de capilaridade, distribuindo por canais vasculares ao resto do corpo, isso comprova a preferência desses animais em habitar lugares úmidos e de baixa insolação como embaixo de troncos, pedras e folhas (Araújo, 1994).

O habitat dos crustáceos terrestres é encontrado em todos os continentes, em paisagem de florestas temperadas e tropicais e cavernas, há registros de mais de 3637 espécies no mundo, sendo 112 no Brasil, e 27 espécies no Rio Grande do Sul. Algumas espécies como Armadillidium vulgare, Armadillo spp. e Porcelio scaber, são as mais abundantes, estando presentes em variados locais do globo terrestre devido a sua capacidade de viver em diversos habitats, sendo sua distribuição uma importante ferramenta para estudo (Araújo et al., 1996; Paoletti e Hassall, 1999); Schmalfuss, 2003; Hassal et al., 2006).

Segundo Loureiro et al. (2006), os indivíduos da sub-ordem Oniscidea são extremamente sensíveis às mudanças em seu habitat, manejos inadequados na agricultura, como o uso de herbicidas e pesticidas que alteram negativamente a qualidade da serapilheira e consequentemente as taxas de consumo pelos seres detritivoros (Boelter et al., 2009). Outra alteração que está diretamente ligado a densidade e diversidade desses animais, segundo Schmalfuss (1975) é a compactação dos solos que altera as condições naturais como o teor de umidade. Contudo, uma das alternativas para evitar o impacto dessas atividades antrópicas na dinâmica das populações de isópodas terrestres e das demais espécies da fauna do solo é manter fragmentos de matas nativas ou secundárias em sistemas agropastoris, com o intuito de preservar as condições naturais ou similares para a sobrevivência desses animais (Skorupa, 2003). Assim, esse trabalho foi conduzido com o objetivo de averiguar a 
importância das reservas legais como refúgio e habitat funcional para populações de isópodas terrestres em áreas agrícolas.

Quadros (2010), afirma que a revegetação de áreas degradadas é uma importante estratégia que permite aos isópodas terrestres desempenhar seu papel na ciclagem de nutrientes. Isto reforça a ideia da importância das áreas de preservação legal como um importante refúgio para esse grupo de organismos.

\section{MATERIAL E MÉTODOS}

A área de estudo localiza-se no município de Santa Maria, região central do estado do Rio Grande do Sul, em uma faixa de terra entre o Planalto Médio e a Depressão Central. A área de estudo abrange a região Nordeste do Município nas coordenadas $29^{\circ} 35^{\prime} 26,02^{\prime \prime}$ de Latitude Sul e $53^{\circ} 41^{\prime} 4,92^{\prime}$ 'Longitude Oeste, ocupando uma área territorial de $0,15 \mathrm{~km}^{2}$. O município está inserido em duas sub-bacias hidrográficas, a do Rio Soturno e do Rio VacacaíMirim, ambas pertencentes ao sistema Jacuí-Guaíba.

O relevo apresenta acentuada ondulação e o tipo de solo predominante é o Neossolo Litólico (Viera e Rovani, 2009) que têm como características serem solos jovens, tendo horizonte superficial assentado sobre rocha alterada ou inalterada, com sequência de horizonte A - R, e em alguns casos A - C - R. A vegetação é formada por floresta subtropical subcaducifólia e por vegetação secundária (Viera e Rovani, 2009). feijão.

A atividade econômica local é agrícola, predominando a plantação de batatas, milho e

O clima de Santa Maria é do tipo, temperado mesotérmico e úmido (Cfa de Koeppen), e a precipitação anual varia em torno de 1500 a 2000 mm (Viera e Rovani, 2009).

Foram amostradas três áreas de preservação ambiental, constituída por mata nativa, e quatro áreas de diferentes cultivos agrícolas (Tabela 1).

Tabela 1. Uso e ocupação do solo das áreas de estudo com as respectivas áreas totais.

\begin{tabular}{lc}
\hline Uso e Ocupação do Solo & Área total $\left(\mathrm{m}^{2}\right)$ \\
\hline Mata Feijão & $24.706,45$ \\
Cultura de Feijão & $6.400,77$ \\
Mata Azevem-Milho & $1.083,32$ \\
Cultura de Azevem & $53.607,07$ \\
Cultura de Milho & $23.106,6$ \\
Mata Soja & 486,27 \\
Cultura de Soja & $39.158,42$ \\
\hline
\end{tabular}

As áreas de mata foram escolhidas de acordo com os tipos de cobertura vegetal, adjacentes (cultivos), que são: azevem, feijão, milho e soja, denominados Mata Azevem-Milho (divisa com dois tipos de plantio), Mata Feijão, Mata Milho e Mata soja. As distribuições e localizações dos locais de estudo são apresentadas na Figura 1. 


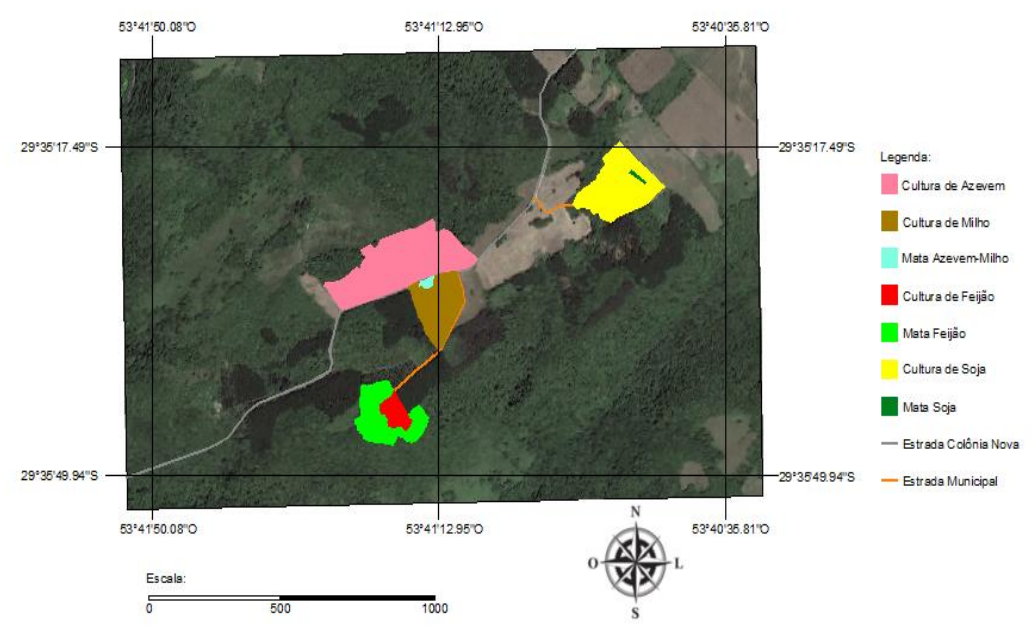

Figura 1. Distribuição dos locais de estudo.

Fonte: adaptado de Google Earth (2013).

Foram amostrados três pontos aleatórios em cada uma das três áreas de mata preservada (Mata Feijão, Mata Azevem-Milho e Mata Soja) e três pontos aleatórios em cada uma das quatro coberturas vegetais (azevem, feijão, milho e soja), sem repetição.

Os pontos de amostragem dos isópodas foram determinados conforme esquema com distribuição estratificada, fazendo-se uma divisão regular da área e distribuindo-se os pontos de forma aleatória em cada uma delas. Com esse esquema de amostragem assegurou-se que todas as subáreas que compõem o local investigado foram amostradas.

Para a coleta dos isópodas foi utilizado uma adaptação do método proposto por Paoletti e Hassal (1999), no qual um quadro de metal medindo $40 \mathrm{~cm}$ x $40 \mathrm{~cm}$ foi cravado no solo numa profundidade de aproximadamente $10 \mathrm{~cm}$ e coletado todo material constituído por serapilheira, fauna e solo, tomando o cuidado para que todo o conteúdo do perímetro do quadrado fosse coletado. O material destinado à captura dos isópodas, constituído de serrapilheira e solo subsuperficial $(0-5 \mathrm{~cm})$, foi acondicionado em sacos de polietileno com furos para permitir a troca de gases.

As amostras foram conduzidas ao laboratório, onde foi realizada a catação manual, contagem e preservação dos exemplares em álcool $75 \%$, para posterior identificação.

A abundância foi estimada pelo número médio de indivíduos encontrados em cada amostra, extrapolado para $\mathrm{m}^{2}$ (Dengerfield, 1990).

A densidade foi obtida pela contagem do número de indivíduos capturados na área total do quadrado de metal e esse número de isópodas foi extrapolado por metro quadrado (ind. $/ \mathrm{m}^{2}$ ).

Logo após a determinação da abundância os isópodas foram fotografados em lupa trinocular marca Meiji (XL50) com câmara digital Sony 5.3 Megapixels, acoplada ao microscópio. Os taxons foram identificados por comparação das imagens e descrições em Paoletti e Hassal (1999) e Pérez-Schultheiss (2010).

A coleta de solo para a determinação do conteúdo de água e a porcentagem de matéria orgânica foi realizada nos mesmos pontos de coleta dos isópodas, removendo-se aproximadamente $500 \mathrm{~g}$ de solo a uma profundidade de $0 \mathrm{~cm}$ a $10 \mathrm{~cm}$ nos locais de mata preservada e áreas cultivadas. O conteúdo de água foi determinado conforme a NBR 6457 (ABNT, 1986) de Preparação para Ensaios de Compactação e Ensaios de Caracterização, anexo: Determinação do Teor de Umidade do solo. A porcentagem de matéria orgânica foi determinada pelo método de Walkley-Black: Titulação após Oxiredução por Via Úmida proposta por Tedesco et al. (1995). E os valores de $\mathrm{pH}$ foram obtidos pelo método de Diluição em Água proposto em Tedesco et al. (1995). 


\section{RESULTADOS E DISCUSSÃO}

Os valores de abundância se referem ao total do número de exemplares de isópodas (crustáceos terrestres) encontrados nos três pontos amostrais de cada área de estudo, e estão expostos na Tabela 2, juntamente com a estimativa de densidade de indivíduos por metro quadrado, e numero de espécies encontradas em cada área. Foram inseridos também, os valores dos parâmetros analisados das amostras de solo.

Como pode ser observado, os únicos locais onde foram encontrados crustáceos terrestres foram nas duas áreas de mata preservada, Mata feijão e Mata Azevem-Milho. Esse resultado pode ser explicado por essas matas apresentarem as condições ideais para o crescimento e desenvolvimento dos isópodas como, temperatura, umidade, quantidade e qualidade de matéria orgânica, ou seja, variabilidade de serrapilheira e palatibilidade da mesma.

Esse resultado corrobora com os obtidos por Boelter et al. (2009), que comprovaram por meio de ensaios em laboratório a preferência de isópodas terrestres por serrapilheira em estágio de 3 a 4 meses de decomposição, mas também completam sua dieta alimentando-se de folhagens verdes. E essas características de alimento são comuns em áreas de mata pela variada composição dos resíduos vegetais.

Tabela 2. Estimativa da abundância, densidade (Dc) e diversidade de isópodes terrestres bem como o conteúdo gravimétrico de água no solo, matéria orgânica (MO) e pH do solo em três fragmentos de mata preservada e quatro áreas cultivadas no município de Santa Maria, RS no outono de 2013.

\begin{tabular}{lcccccc}
\hline Local de estudo & Ug* $(\%)$ & $\mathrm{pH}$ & $\begin{array}{c}\mathrm{MO} \\
\left(\mathrm{gkg}^{-1}\right)\end{array}$ & Abundância & $\begin{array}{c}\text { Dc } \\
(\text { ind./m² }\end{array}$ & $\begin{array}{c}\text { Diversidade } \\
\text { de isópodes }\end{array}$ \\
\hline Mata Feijão & 42,14 & $7,1-7,2$ & 57,23 & 28 & 58,33 & 2 \\
Cultivo de Feijão & 32,68 & 5,32 & 28,14 & 0 & 0 & 0 \\
Mata Azevem - Milho & 28,7 & 5,65 & 43,78 & 15 & 31,25 & 1 \\
Cultivo de Azevem & 28,32 & 5,12 & 28,96 & 0 & 0 & 0 \\
Cultivo de Milho & 34,97 & 5,28 & 29,13 & 0 & 0 & 0 \\
Mata Soja & 36,7 & 5,43 & 29,48 & 0 & 0 & 0 \\
Cultivo de Soja & 34,26 & 5,29 & 28,11 & 0 & 0 & 0 \\
\hline
\end{tabular}

Nota:* Conteúdo gravimétrico (porcentagem de água em 100 g de solo).

Pela palatibilidade, ocorre a preferência desses animais por resíduos vegetais com baixo teor de tanino, assim, em uma mata pode escolher por um tipo de vegetação (Boelter et al., 2009). A mata adjacente a lavoura de soja não foi encontrado nenhum exemplar, isso pode ser decorrente de um efeito de borda, como uma interferência das atividades agrícolas.

Ao contrário, nas áreas cultivadas adjacentes às matas, não foi encontrado nenhum crustáceo terrestre, essas áreas apresentam reduzida variabilidade de serrapilheira pelo plantio de monoculturas, e o uso de insumos agrícolas alteram a qualidade dos resíduos vegetais que segundo Paoletti e Hassall (1999) alteram a atividade detritiva desses animais e ocasionando a mortalidade dos mesmos.

Quanto à densidade os resultados evidenciaram um número considerado de indivíduos por $\mathrm{m}^{2}$ em duas das áreas de mata amostradas, comparado aos números encontrados por Paoletti e Hassal, numa investigação realizada em 1999 na Holanda, onde foram encontrados mais de 200 ind. $/ \mathrm{m}^{2}$ em áreas de mata, e uma média de 33,1 ind. $/ \mathrm{m}^{2}$ em áreas agrícolas, onde o uso de agrotóxicos não é intenso como no Brasil. Pode-se afirmar que a diminuição da densidade no local de estudo remete às observações feitas em relação à abundância, ou seja, 
muito provavelmente seja devido ao reduzido número de indivíduos que conseguiram sobreviver ao manejo inadequado das áreas cultivadas e quem sabe, ainda, um possível efeito de borda nas matas já que estas não são tão extensas.

As análises de solo evidenciaram que a área da Mata Feijão é que apresenta as melhores características para a sobrevivência dos crustáceos terrestres, como bons teores de matéria orgânica $\left(57,23 \mathrm{gkg}^{-1}\right)$, conteúdo de água $(42,14 \%)$ e $\mathrm{pH}(7,1-7,2)$. Isso é evidenciado por Paoletti e Hassall (1999), que estimaram a ocorrência de numero significantemente maior de isópodes em florestas úmidas e com teores de matéria orgânica mais elevada do que em áreas agricultáveis, mais degradadas pela atividade agropastoril. Em termos de pH, solos mais básicos ocorre uma maior adsorção de cálcio à argila, e os crustáceos terrestres necessitam de cálcio para a formação do exoesqueleto, conforme relatado em Paoletti e Hassall (1999), habitando solos mais básicos, isso justifica porque foram encontrados mais exemplares em mata com $\mathrm{pH}$ mais elevados.

No local Mata Azevem-Milho, onde foram encontrados 15 exemplares de crustáceos terrestres, os parâmetros analisados de solo não foram muito diferentes dos de lavoura, exceto os teores de matéria orgânica $\left(43,78 \mathrm{gkg}^{-1}\right)$. Que pode ser explicada pela atividade desses organismos nessa mata, pois, mesmo apresentando valor de umidade e $\mathrm{pH}$ próximos ao encontrado nas lavouras, os resíduos vegetais apresentam variabilidade e qualidades suficientes para sua sobrevivência. Pode se concluir que essa mata está sofrendo um efeito de borda ocasionado pelas lavouras de azevem e milho.

Comparando os parâmetros de matéria orgânica e pH analisados dos solos dos locais de estudo com os parâmetros observado por Alho et al. (2007), para Neossolos Litólicos, pode-se observar uma queda desses valores para Neossolo Litólico de Silveira Martins, enquanto o pesquisador citado descreveu valores de matéria orgânica entre $92,80 \mathrm{gkg}^{-1}$ e $34,40 \mathrm{gkg}^{-1} \mathrm{em}$ condições normais, nos solos da área em estudo a matéria orgânica variou de $34,2 \mathrm{gkg}^{-1}$ (áreas de mata) a $28,11 \mathrm{gkg}^{-1}$, evidenciando que a atividade agrícola na região está descaracterizando de forma negativa os solos. O pH normal de um Neossolo Litólico segundo Alho et al. (2007) fica entre uma faixa de 7,4 e 6,3, e os obtidos por meio de ensaio dos locais de estudo ficaram entre uma faixa de 7,2 (áreas de mata) a 5,12, este último valor muito mais ácido que os valores normais para esse solo, também decorrentes dos impactos negativos das atividades agrícolas.

A diversidade de espécies encontrada nesta investigação está muito aquém do esperado para áreas de mata onde, o observado, há boas condições de nutrientes, temperatura e umidade. Este fato deve-se à pressão de seleção imposta pelo manejo das áreas de entorno que, além de eliminar muitas espécies que não possuem capacidade de suportar as alterações do seu habitat, são prejudicadas pela competição com as espécies exóticas que, ao contrário, são favorecidas. Para justificar esta inferência pode-se observar que as duas únicas espécies encontradas nas áreas de mata amostradas não são nativas e sim, exóticas, originárias da Europa. Também se observa que o encontro da mesma espécie em dois fragmentos de mata remete a proximidade da Mata Feijão com a Mata Azevem-Milho.

\subsection{Descrição das espécies encontradas:}

Armadillidium nasatum (Budde-Lund, 1985)

Tamanho: $4 \mathrm{~mm}$

Urópodes largos e curtos, alinhados à extremidade final do abdômen; cabeça com um pequeno nódulo central em forma de $\mathrm{V}$ e projeção nitidamente quadrada entre as antenas; télson nitidamente quadrangular; segundo par de antenas possui flagelum com 2 artículos; superfície do corpo e cabeça apresentando saliências semelhantes à tubérculos, contrátil em forma de bola, com uma listra central de coloração marrom-escuro e listras laterais amarelo- 
claro; cinco pares de pseudotraquéias; um par de olhos pequenos e compostos (Figura 2a e 2b).

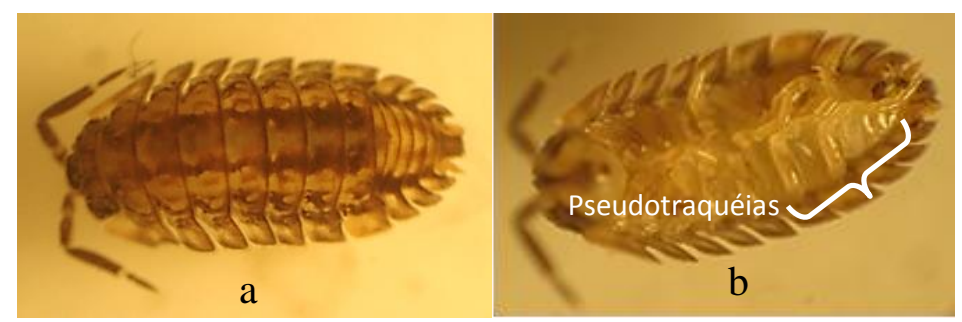

Figura 2. Vista dorsal evidenciando a forma quadrangular do télson, projeção quadrada no cefalotórax, curvatura do corpo e capacidade volvacional (a). Vista ventral ressaltando os cinco pares de pesudotraquéias (b).

Philoscia muscorum muscorum (Scopoli, 1763)

Tamanho: $6 \mathrm{~mm}$ (11 mm conforme literatura especializada).

Urópodes estreitos e estendidos além da extremidade do abdômen e levemente inclinados para cima do corpo; Cabeça desprovida de lobos, de cor preta, mais escura que o corpo, e com pequenas manchas amarelas. Segundo par de antenas de coloração marrom com estrias amarelas e com flagelum composto por 3 artículos; Abdômen abruptamente mais estreito que o tórax e télson nitidamente pontudo; corpo estreito, amarelo com marrom e uma linha mediana escura (Figura 3 a, 3 b e $3 c$ ).
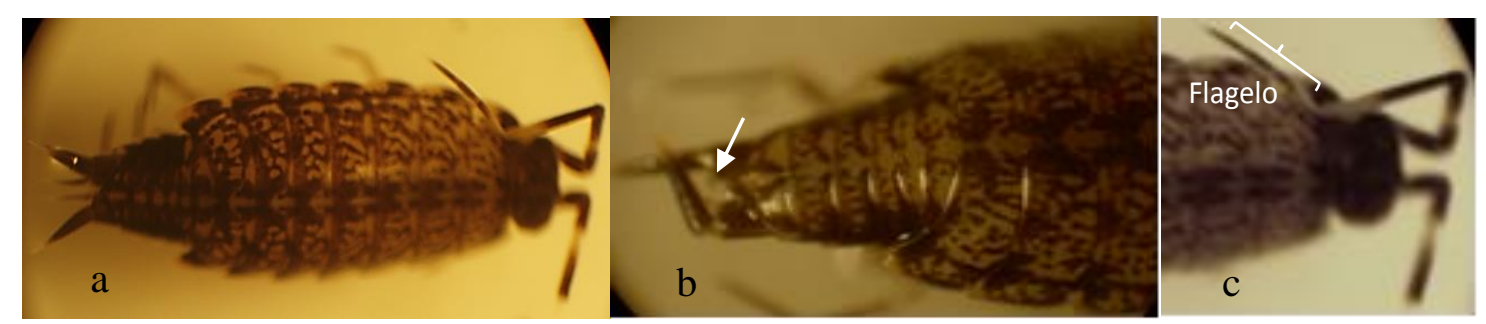

Figura 3. Morfologia da cabeça, abdômen e urópodes de Philoscia muscorum (a), forma do télson (b) e número de artículos do flagelum do segundo par de antenas (c).

A espécie de crustáceo terrestre ilustrada na Figura 3 mede entre $6 \mathrm{~mm}$ de comprimento, possui corpo achatado e cabeça em forma de escudo. O corpo é dividido encefalotórax, subdividido em 7 segmentos distintos e abdômen cujos segmentos encontram-se fundidos com télson. $\mathrm{O}$ aparelho bucal é formado por peças bucais compactas, que formam uma massa bucal prognata (com as mandíbulas proeminentes), protegida por apêndices especializados.

\section{CONCLUSÃO}

As reservas legais dos agroecossistemas são fundamentais para a conservação de isópodes, mas também como um refúgio vital para a fauna do solo de todo o seu entorno.

As áreas agricultáveis, independente do tipo de cobertura vegetal, alteram a diversidade de isópodas que desempenham importante função na decomposição de matéria orgânica e bioacumulação de metais pesados. 


\section{AGRADECIMENTOS}

Os autores agradecem ao Centro Universitário Franciscano pelo apoio financeiro nas saídas a campo, para coleta de amostras bem como pela disponibilização dos laboratórios para o desenvolvimento da pesquisa.

\section{REFERENCIAS}

ALHO, D. R. et al. Caracterização física, química e mineralógica de Neossolos Litólicos de diferentes materiais de origem. Revista Brasileira de Ciências Agrárias, Recife, v. 2, n. 2, p.117-122, abr.jun., 2007.

ARAUJO, P. B. Isópodos: os crustáceos colonizadores da terra. Acta Biologica Leopoldensia, v. 16, p. 15-27, 1994.

ARAUJO, P. B. et al. Isópodos terrestres de Santa Catarina e Rio Grande do Sul (Crustacea, Oniscidea). Iheringia, v. 81, p. 111-118, 1996.

ASSOCIAÇÃO BRASILEIRA DE NORMAS TÉCNICAS - ABNT. NBR 6457. Amostras de solo: preparação para ensaio de compactação e ensaios de caracterização. Rio de Janeiro, 1986.

BOELTER, J. F. et al. The feeding rates and preferences of a neotropical terrestrial isopod (Oniscidea). Nauplius, v. 17, n. 2, p. 107-113, 2009.

CASEIRO, I.; SANTOS, S.; SOUSA, J. P.; NOGUEIRA, A. J. A.; SOARES, A. M. V. M. Optimization of culture conditions of Porcellio dilatatus (Crustaceae, Isopoda) for laboratory teste development. Ecotoxicology and Environmental Safety, v. 47, p. 285$291,2000$.

DENGERFIELD, J. M. Competition and the Effects of Density on Terrestrial Isopods. Zoology, v. 4, p. 411-423, 1990.

FURLAN, S. A. Indicadores Biogeográficos em fragmentos de Mata Atlântica insular e continental e suas possíveis implicações paleoambientais. Revista do Departamento de Geografia, São Paulo, n. 10, p. 13-28, 1996.

GARCIA, F. R. M.; CAMPOS, J. V. Biologia e controle de artrópodes de importância fitossanitária (diplopoda, symphyla, isopoda), pouco conhecidos no Brasil. Biológico, São Paulo, v. 63, n.1/2, p. 7-13, jan./dez., 2001.

GRÜNWALD, M. Adaptation und Dekompostierungsleistung von Landasseln (Isopoda, Oniscidea) an Standorten der großen Brennessel (Urtica dioica L.). Hochschulsammlung Naturwissenschaft, Biologie, Freiburg, v. 20, p. 1-273, 1988.

HASSALL, M. et al. Biodiversity of terrestrial isopods along a gradient of disturbance in Sabah, East Malaysia. European Journal of Soil Biology, v. 42, p. 197-207, 2006. http://dx.doi.org/10.1016/j.ejsobi.2006.07.002

LOUREIRO, S. et al. Feeding behaviour of the terrestrial isopod Porcellionides pruinosus Brandt, 1833 (Crustacea, Isopoda) in response to changes in food quality and contamination. Science of Total Environment, v. 369, p. 119-128, 2006. http://dx.doi.org/10.1016/j.scitotenv.2006.05.023 
PAOLETTI, M. G.; HASSALL, M. Woodlice (Isopoda: Oniscidea): their potential for assessing sustainability and use as bioindicators. Agriculture, Ecosystems and Environment, v. 74, p. 157-165, 1999. http://dx.doi.org/10.1016/S01678809(99)00035-3

PÉREZ-SCHULTHEISS, J. Famílias de isópodos terrestres (CRUSTACEA: ISOPODA: ONISCIDEA) de Chile: sinopsis y clave de identificación. Boletín de Biodiversidad de Chile, v. 4, p. 63-82, 2010.

QUADROS, A. F. Os isópodos terrestres são boas ferramentas para monitorar e restaurar áreas impactadas por metais pesados no Brasil? Oecologia Australis, v. 14, n. 2, p. 569-583, 2010.

RABATIN, S. C.; STINNER, B. R. Indirect effects of interactions between VAM fungi and soil-inhabiting in vertebrates on plant processes. Agriculture Ecosystems \& Environment, v. 24, p. 135-146, 1988.

RUPPERT, E. E.; FOX, R. S.; BARNES, R. D. Zoologia de invertebrados: uma abordagem funcional evolutiva. 7. ed., São Paulo: Roca, 2005. 1.145 p.

SCHMALFUSS, H. World catalog of terrestrial isopods (Isopoda: Oniscidea). Stuttgarter Beitragezur Naturkunde Serie A, v. 654, p. 341, 2003.

SCHMALFUSS, H. Morphologie, Funktion der tergalen Langsrippen bei Lãnd isopodan (Oniscoidea, Isopoda, Crustacea). Zoomorphologie, v. 86, p. 155-157, 1975.

SKORUPA, L. A. Áreas de preservação permanente e desenvolvimento sustentável. Jaguariúna: EMBRAPA Meio Ambiente, 2003. 4p.

TEDESCO, M. J. et al. Análise de solo, plantas e outros materiais. Porto Alegre: UFRGS, 1995. 174p.

VIERA, E. G.; ROVANI, F. F. M. O uso da terra em Silveira Martins: uma nova perspectiva ou possibilidade por meio das geotecnologias. Santa Maria: UFSM, 2009. Disponível em: 〈www.egal2009.easyplanners.info/area04/413 >. Acesso em: 20 nov. 2013. 